\title{
Effects of High-Velocity Strength Training on Movement Velocity and Strength Endurance in Experienced Powerlifters with Cerebral Palsy
}

\author{
by \\ Rafał Szafraniec ${ }^{1}$, Aleksandra Kisilewicz', Martyna Kumorek1, Mathias Kristiansen², \\ Pascal Madeleine ${ }^{2}$, Dariusz Mroczek ${ }^{3}$
}

The present study aimed to evaluate the effects of a 6-week high-velocity strength training (HVST) intervention on movement velocity and strength endurance in experienced powerlifters with cerebral palsy (CP). Eleven experienced powerlifters with CP and seven from a control group (CON), were subjected to 6-week HVST. An assessment of movement velocity and strength endurance was conducted one week before (T1) and one week after (T2) the 6-week training intervention. During testing, athletes performed a maximum number of bench press repetitions possible within 5 sets of $15 \mathrm{~s}$ each, with 1-min passive rest intervals in-between. The indicator of movement velocity was the weight pressed in the first $5 \mathrm{~s}(5 \mathrm{sW})$ in all performed sets. Strength endurance was described by the total weight (TW) pressed during the test. $5 \mathrm{sW}$ in $\mathrm{T} 2$ was significantly higher as compared with T1 in the CP group only (T1 $928.9 \pm 342.9 \mathrm{~kg}$ vs. T2 1007.3 $\pm 324.6 \mathrm{~kg} ; p=0.016$ ). TW in T2 was significantly higher as compared with T1, both in the CP group (T1 $2550.5 \pm 843.9 \mathrm{~kg}$ vs. T2 $2809.8 \pm 981.3 \mathrm{~kg} ; \mathrm{p}<0.001)$ and in the CON group (T1 $2300.7 \pm 845.1 \mathrm{~kg}$ vs. T2 $2468.9 \pm$ $890.1 \mathrm{~kg} ; p=0.049)$. A 6-week program of HVST increased movement velocity in resistance trained CP athletes. The gains of strength endurance were observed in both groups.

Key words: performance, disabled athletes, resistance exercise, resistance.

\section{Introduction}

According to the rules of the International Paralympic Committee, the paralympic powerlifting discipline is open to male and female athletes being at least 14 years of age, who possess impairment in muscle and joint function (power, range of motion and hypertonia), movement deficiencies (ataxia and athetosis), differences in physical structure (limb deficiency, leg length discrepancy and short stature), and/or a range of physical disabilities (cerebral palsy, spinal cord injuries, lower limb amputation, and poliomyelitis). All eligible athletes compete in one sport class, although in different weight categories. The bench press itself is a single discipline in paralympic powerlifting, in which athletes must be able to lower the bar to the chest level, hold it motionless on their chest, and then press the bar upwards, with the arms straight and elbows locked, with no more than a $20^{\circ}$ loss of full extension of either elbow permitted.

Athletes with $\mathrm{CP}$ form a special group of competitive athletes in paralympic powerlifting. $\mathrm{CP}$ is a complex disorder in which motor impairment is often accompanied by disturbances in sensation, perception, cognition, communication, and behaviour (Schiariti et al., 2015). Klenck and Gebke (2007) defined CP as impairment of movement and posture due to damage to an area or areas of the brain that

\footnotetext{
1 - Department of Paralympic Sports, University School of Physical Education in Wroclaw, Poland.

2 - Sport Sciences - Performance and Technology, Department of Health Science and Technology, Aalborg University, Aalborg, Denmark.

3 - Department of Biological and Motor Sport Bases, University School of Physical Education in Wroclaw, Poland.
} 
control and coordinate muscle tone, reflexes, posture, and movement. Individuals with $\mathrm{CP}$ are typically characterized by a lower muscle mass and deficits in both strength and the production of higher velocity movements, i.e., muscle power (Moreau et al., 2013). Poor coordination due to reduced agonist muscle activation and changes in patterns of co-activation is another very important disadvantage (Hussain et al., 2014). In turn, high performance in a strength task requires the proper activation and timing of all agonist, synergist and antagonist muscles (Kristiansen et al., 2016). Limitations in this population are likely to result from coordination difficulties or as a result of the impaired rate of force development considering maximal force production (Beckman et al., 2017; Chiu et al., 2010). This may hamper task performance especially when repetitive contractions are involved. Thus, it has been shown that both the laboratory repetitions-tofatigue protocol and the clinical squat test provide evidence for reduced ability in patients with $\mathrm{CP}$ to generate repetitive movements, in comparison to their healthy peers (Eken et al., 2014, 2017). Despite poor coordination, reduced movement velocity and the impaired rate of force development, athletes with $\mathrm{CP}$ compete with athletes with unaffected upper limb function. For $\mathrm{CP}$ powerlifters to successfully compete with other powerlifters, training should focus on improving movement velocity and peak power development, even though the velocity component of muscle contraction is neglected in traditional strength training. However, GonzálesBadillo et al. (2014) claim that movement velocity can be considered a fundamental component of resistance training intensity, since, for a given relative intensity, the velocity at which loads are lifted largely determines the training effect, and bench press strength gains can be maximized when repetitions are performed at maximal intended velocity. Most of the studies examining the effect of movement velocity on neuromuscular performance have been conducted on isokinetic equipment, but only a few have used isoinertial (constant external load) exercise as a training modality (González-Badillo et al., 2014). Moreau et al. (2013) stated that HVST was equally effective as traditional strength training in improving isokinetic strength of the knee extensors, but more effective in improving the velocity of movement and muscle power in $\mathrm{CP}$ patients.

The aforementioned works concerned the effectiveness of HVST either in a healthy population (González-Badillo et al., 2014) or a CP youth population (8-20 years old) (Moreau et al., 2013) and referred to the lower limbs. To the best of our knowledge, no studies have analysed HVST application in well-trained adults with CP. Therefore, this study aimed to evaluate the effects of a 6-week HVST intervention on movement velocity and strength endurance in experienced powerlifters with $\mathrm{CP}$. Based on the facts presented above, we expected an improvement in movement velocity and strength endurance in $\mathrm{CP}$ athletes. We also hypothesized that the improvement would be smaller as compared to powerlifters with unaffected motor coordination in upper limbs .

\section{Methods}

\section{Participants}

The study included 21 male disabled powerlifters. Only 18 of the participants completed the entire study. For eligibility, participants were required to have a minimum of 3 years of powerlifting experience. The exclusion criteria were injuries within the last 6 months or a break in training ( $>1$ week in duration) in the last 3 months. Participants with cerebral palsy formed the $\mathrm{CP}$ group $(\mathrm{n}=11)$, and disabled powerlifters with unaffected motor coordination in upper limbs were classified to the control group (CON; $n$ $=7$ ). The characteristics of the participants are summarized in Table 1.

The study was approved by the local ethics committee and participants provided written informed consent after receiving verbal and written information on the experimental protocol.

\section{Experimental design}

A repeated measures design was employed with two independent groups (CP and $\mathrm{CON}$ ). The movement velocity and strength endurance were measured during the bench press exercise with increased loads. First of all, such a testing method can be used in a typical training environment, which is important when evaluating a population with disabilities, because it eliminates the disadvantages associated with transport to the laboratory. Secondly, testing 
involves performing their basic exercise, i.e. bench press. One week before testing, body mass and a one-repetition maximum (1RM) load were evaluated (Ritti-Dias et al., 2011).

Movement velocity and strength endurance testing

Immediately before testing, participants completed a general warm-up that included dynamic exercises and stretching of the shoulders and upper limbs, followed by a specific warm-up consisting of 3 sets $\times 12$ repetitions of the barbell bench press with a load of 30\% 1RM. After the warm-up, participants assumed a lying position on the bench with thighs stabilized using a belt, as per the regulations of the International Powerlifting Federation. An official powerlifting bench and barbell (Eleiko Sport AB, Halmstad, Sweden) were used during the test. The barbell was moved from the rack to an athlete by a platform service. A signal to start the attempt was given once an athlete had held the bar with the upper limbs in extension. Then, athletes were asked to perform the maximum number of repetitions, as fast as possible, within 5 sets of $15 \mathrm{~s}$ each, with 1-min passive rest intervals between sets. Only complete cycles of lowering the bar while touching the chest and pressing the bar up to full elbow extension were recorded. Besides, bouncing the bar of the chest was not allowed. The load was progressively increased between sets, starting at an initial load of approximately $40 \% 1 \mathrm{RM}$ and progressing by $10 \% 1 \mathrm{RM}$ during successive attempts.

In this way, participants worked with a load they usually used during a training unit (40$80 \%$ 1RM). The same absolute load was used in both testing sessions: one week before (T1) and one week after (T2) the 6-week HVST program. The number of repetitions completed both in the first $5 \mathrm{~s}$ and in $15 \mathrm{~s}$ of each set was recorded. Then, the weight pressed in the first $5 \mathrm{~s}(5 \mathrm{sW})$ of all sets and the total weight (TW) pressed during the test were calculated as follows:

$5 \mathrm{sW}$ set $(\mathrm{kg})=$ load $(\mathrm{kg}) \times$ number of repetitions in first $5 \mathrm{~s}$ of the set

$5 \mathrm{sW}=5 \mathrm{~s} W_{\text {set1 }}+5 \mathrm{~s} W_{\text {set2 } 2}+5 s W_{\text {set3 }}+5 s W_{\text {set4 }}+5 s W_{\text {set5 }}$ $\mathrm{TW}_{\text {set }}(\mathrm{kg})=$ load $(\mathrm{kg}) \times$ number of repetitions in the set

$\mathrm{TW}=\mathrm{TW}_{\mathrm{set} 1}+\mathrm{TW}_{\mathrm{set} 2}+\mathrm{TW}_{\mathrm{set} 3}+\mathrm{TW}_{\mathrm{set} 4}+\mathrm{TW}_{\mathrm{set} 5}$

The $5 \mathrm{sW}$ is a measure of movement velocity and TW indicates strength endurance.
Both testing and training units were supervised by the coach of the national paralympic team in powerlifting to ensure that a complete range of motion and proper technique were used in each successful bench press repetition and the training program was properly implemented.

\section{High-velocity strength training}

The HVST intervention was conducted over 6 weeks, 5 days per week, with each session of approximately 2 hours in duration. The training protocol included a warm-up and specific training, as described below.

A general warm-up included dynamic exercises and stretching of the shoulders and upper limbs and a specific warm-up consisted of the barbell bench press (only the bar: $20 \mathrm{~kg}$ ), which included 3 sets $\times 12$ repetitions. The main part of the training protocol included: $3 \times 8(40 \%$ $1 \mathrm{RM}), 3 \times 6(50 \% 1 \mathrm{RM}), 3 \times 4(60 \% 1 \mathrm{RM}), 3 \times 3(70 \%$ $1 \mathrm{RM}), 3 \times 2(80 \% 1 \mathrm{RM})$, and $3 \times 1(90 \% 1 \mathrm{RM})$. Interset rest intervals ranged from 3 (loads $\leq 60 \% 1 \mathrm{RM}$ ) to 5 min (loads $>60 \% 1 \mathrm{RM}$ ). The concentric phase of bench press exercise was always performed as fast as possible, and the eccentric phase was executed at a slower velocity (i.e., self-selected) than the concentric phase.

\section{Statistical analysis}

All data in the text and figures are presented as mean $\pm \mathrm{SD}$. The normal distribution of the data was examined using a KolmogorovSmirnov normality test. The CP group's performance was compared with the CON group using a mixed-model ANOVA, repeatedmeasures analysis with one between factor (group; $\mathrm{CP}$ and $\mathrm{CON}$ ) and one within factor (time; $\mathrm{T} 1$ and T2). Post-hoc multiple comparisons (Fisher method) were performed if a significant main effect was observed. For each ANOVA, partial eta-squared was calculated as a measure of effect size. Values of $0.02,0.13$, and above 0.26 were considered as a small, medium, and large, respectively (Bakeman, 2005). Statistical analyses were performed using Statistica 13.1 software (Dell, USA). The significance level was set at alpha $<0.05$.

\section{Results}

There were no significant differences between athletes with $\mathrm{CP}$ and $\mathrm{CON}$ in age $(p=$ $0.445)$, body mass $(p=0.281)$, resistance training 
experience $(p=0.305)$ and 1RM value $(p=0.471)$. The intervention and post-assessments were completed in 18 of 21 athletes. Three athletes missed training sessions ( $>2$ weeks) because of injury $(n=2)$ or illness $(n=1)$. The injuries were not related to the applied intervention.

\section{Movement velocity}

Mixed-model ANOVA demonstrated a significant main effect of time $(F=8.12, p=0.011$, $\eta_{\mathrm{p}}{ }^{2}=0.33$ ). Post-hoc comparison revealed that $5 \mathrm{sW}$ in T2 was significantly higher, as compared with $\mathrm{T} 1$, in the CP group (T1 $928.9 \pm 342.9 \mathrm{~kg}$ vs. T2 $1007.3 \pm 324.6 \mathrm{~kg} ; p=0.016$ ) (Figure 1). The improvement of $5 \mathrm{sW}$ was observed in 7 of the 11 athletes. In 2 athletes a decline was observed and in 2 others no change was noticed (Figure 2). The highest increase was $36.3 \%$, and the largest decline was $7.5 \%$. In the CON group $5 \mathrm{sW}$ amounted to $826.1 \pm 346.8 \mathrm{~kg}$ in T1 and $882.1 \pm$ $360.8 \mathrm{~kg}$ in T2 $(p=0.148)$. The main effect of group was non-significant $\left(F=0.49, p=0.495, \eta_{\mathrm{p}}{ }^{2}=0.03\right)$.

\section{Strength endurance}

The mixed-model ANOVA indicated a significant main effect of time $(F=17.79, p<0.001$, partial eta squared $\left.\eta_{\mathrm{p}}{ }^{2}=0.52\right)$. The post-hoc multiple comparison revealed that TW in T2 was significantly higher as compared with $\mathrm{T} 1$, both in the CP (T1 $2550.5 \pm 843.9 \mathrm{~kg}$ vs. T2 $2809.8 \pm 981.3$ $\mathrm{kg} ; p<0.001$ ) and the CON group (T1 $2300.7 \pm$ $845.1 \mathrm{~kg}$ vs. T2 $2468.9 \pm 890.1 \mathrm{~kg} ; p=0.049$ ) (Figure 1).

In the $\mathrm{CP}$ group, TW was greater at $\mathrm{T} 2$ than $\mathrm{T} 1$ in 10 of the 11 athletes, with the highest increase being $21 \%$, and with a decline of $3.9 \%$ noted in 1 athlete (Figure 3). In the CON group, improvement in TW was observed in 6 of the 7 cases, with a maximal increase of $18.8 \%$, and with a decline of $3.0 \%$ observed in one athlete. The main effect of the group was non-significant $(F=$ $0.47, p=0.503, \eta_{\mathrm{p}}{ }^{2}=0.03$ ).

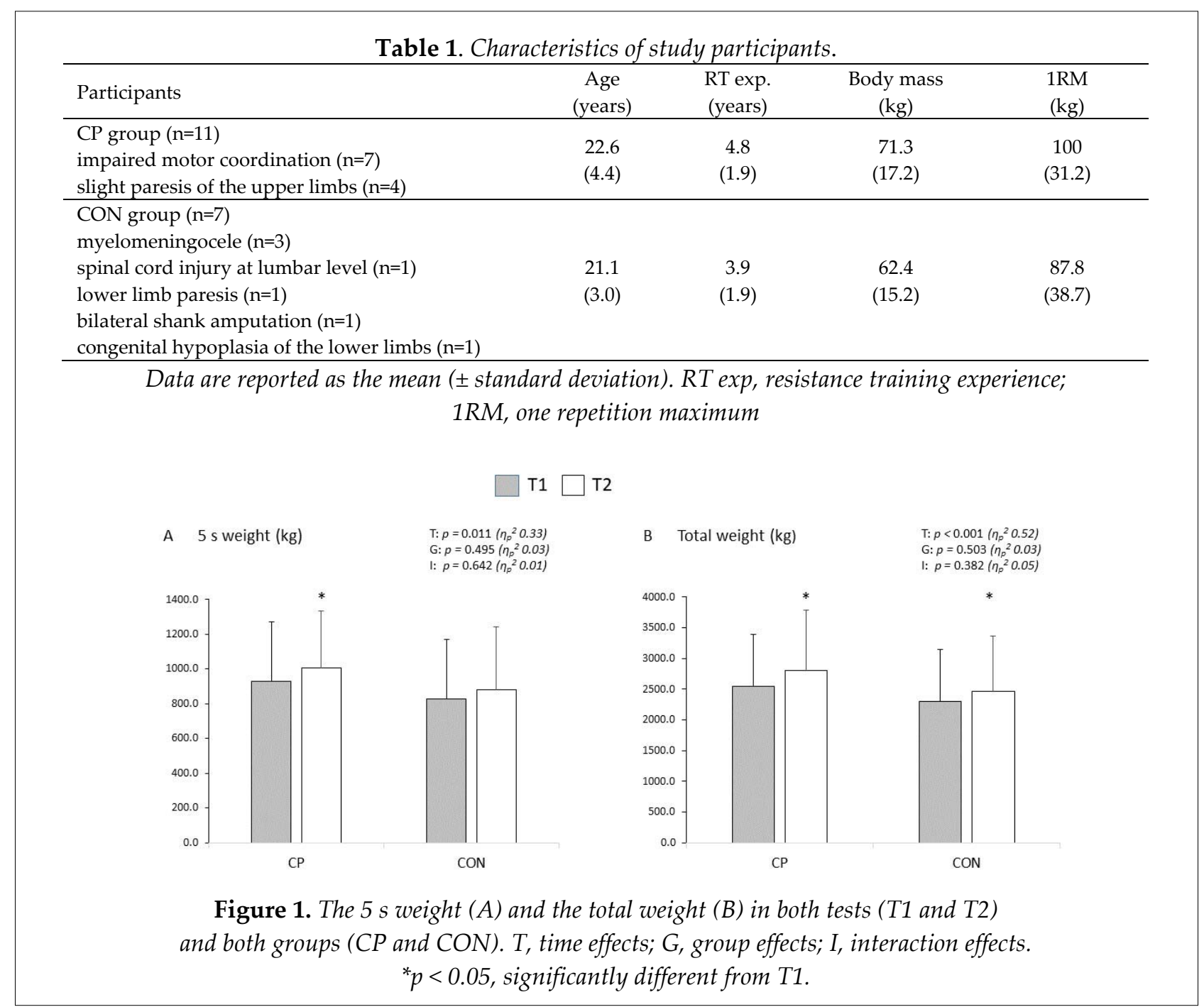




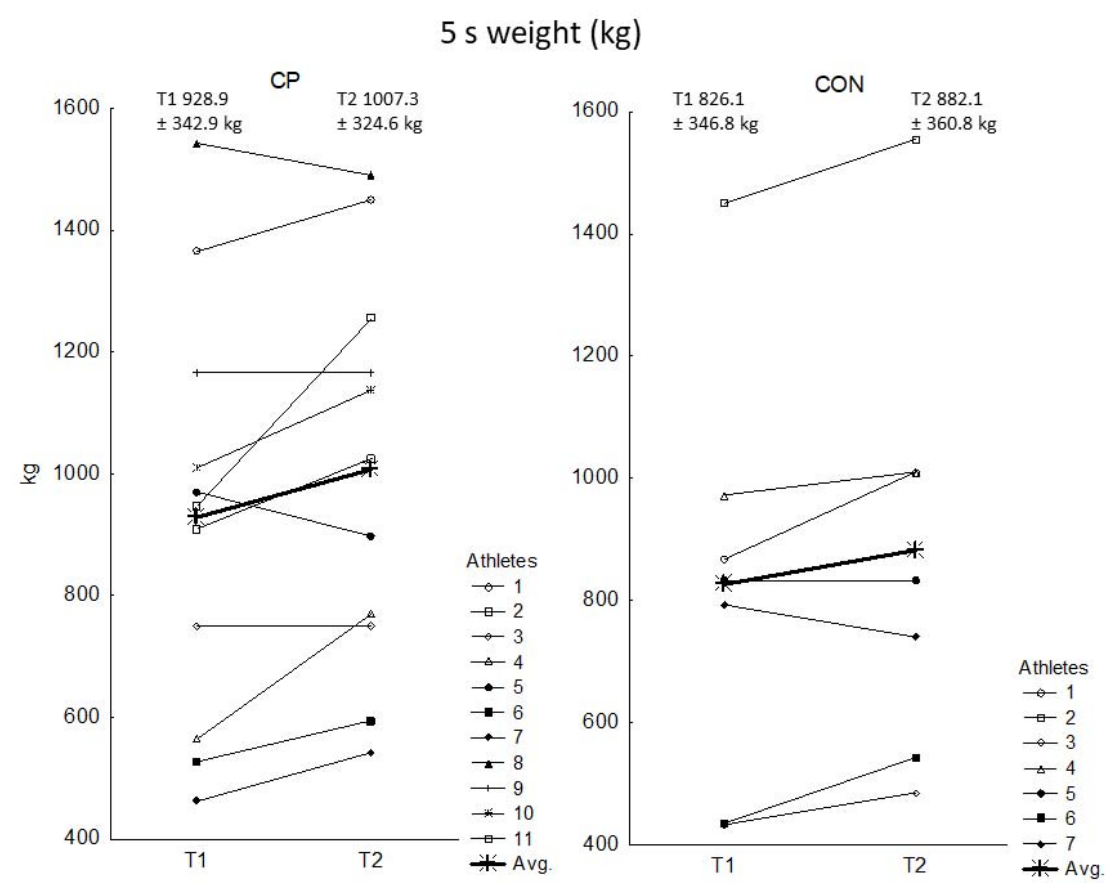

Figure 2. Individual values of the $5 \mathrm{~s}$ weight (5sW) pressed before (T1) and after (T2) the 6-week high-velocity strength training intervention in the CP and CON groups.

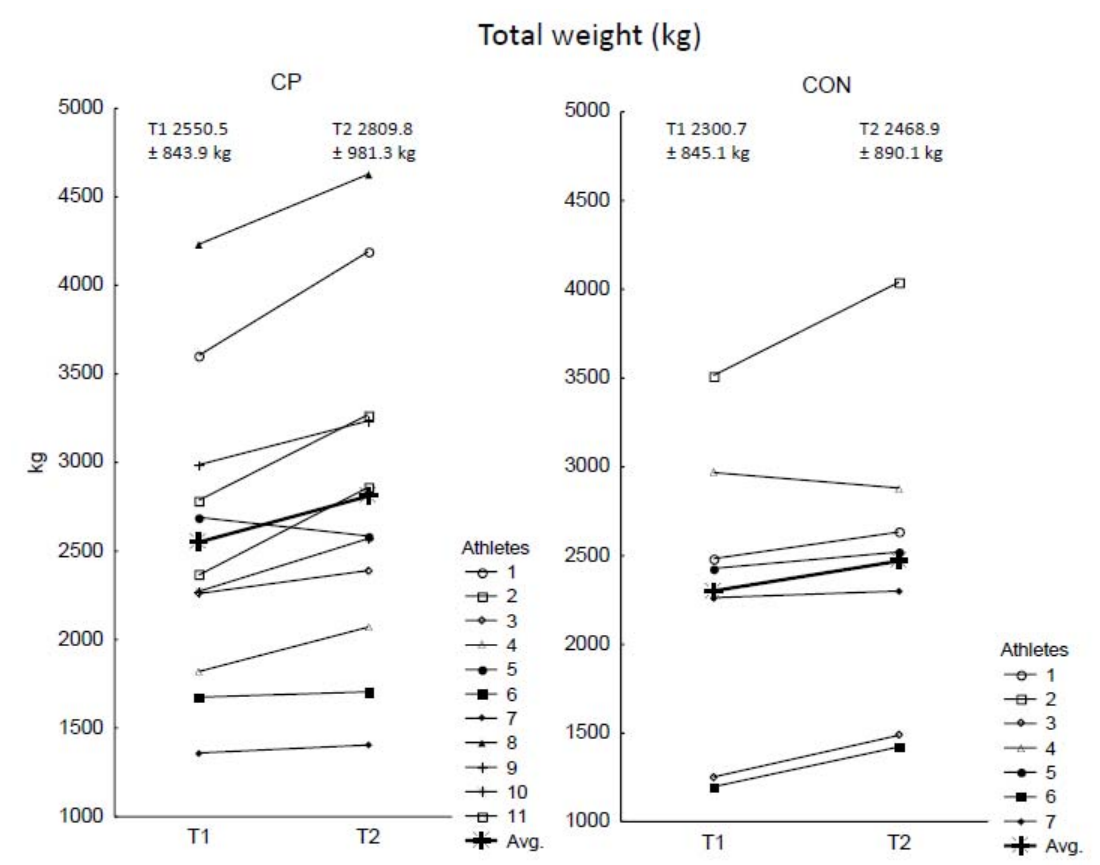

Figure 3. Individual values of total weight (TW) pressed before (T1) and after (T2) the 6-week high-velocity strength training intervention in the CP and CON groups. 


\section{Discussion}

The aim of this study was to evaluate the effects of a high-velocity strength training intervention on movement velocity and strength endurance in experienced powerlifters with cerebral palsy. We hypothesized a gain in movement velocity and strength endurance in $\mathrm{CP}$ powerlifters. Furthermore, we also hypothesized that the increase would be smaller as compared to the group of athletes with unaffected motor coordination in the upper limbs. The results showed that 6 weeks of HVST significantly increased movement velocity in CP powerlifters, however, improvements in strength endurance were observed in both groups. The results confirm our hypothesis that HVST increases movement velocity and strength endurance in well-trained adults with $\mathrm{CP}$. On the other hand, the hypothesis that the gain should be lower in $\mathrm{CP}$ athletes than in subjects with unaffected motor coordination in the upper limbs was not supported.

To the best of our knowledge, no study has previously analysed the effects of HVST on movement velocity and strength endurance in resistance-trained athletes with $\mathrm{CP}$. Therefore, it is not possible to directly compare our data with previous studies. Nevertheless, Moreau et al. (2013) investigated the effects of HVST on muscle architecture and performance, as compared with traditional strength training on youth individuals (age 8-20 years) with CP. They stated that HVST was equally effective as traditional strength training in improving isokinetic strength of the knee extensors, but more effective in improving the velocity of movement and muscle power (Moreau et al., 2013). It is an important fact because it was observed that for $\mathrm{CP}$ patients the average muscle power was only $18 \%$ when strength amounted to $50 \%$ of that measured in typically developing peers (Moreau et al., 2013). These studies support the use of HVST in people with $\mathrm{CP}$, demonstrating its effectiveness in increasing movement velocity and power. This is in line with our results as we found an increase in movement velocity in the $\mathrm{CP}$ group which shows that there was a significant potential to improve this ability. In turn, the lack of significant gain in the $\mathrm{CON}$ group may indicate that their movement velocity was already at the maximal level.

On the other hand, the results of Eken et al. (2014) indicate that adolescents with CP have a reduced ability to endure activity in a submaximal repetitions-to-fatigue test at similar relative loads compared with typically developing adolescents. A lack of differences in TW, observed in our study, between $\mathrm{CP}$ and CON groups, most probably results from the fact that our study involved athletes with several years of experience in strength training, with the majority being successful at the national, European or world championships. Runciman et al. (2015) stated that elite athletes with $\mathrm{CP}$ might have a different exercise response than untrained individuals. Those authors propose that this may result from high intensity training over many years. Another reason for the discrepancy in our results and Eken et al. (2014) may be due to different research methodologies. In the studies of those authors, participants performed three submaximal tests, consisting of a series of isotonic knee extension contractions against a submaximal load until exhaustion. The velocity of movement was slow and amounted to approximately $60 \%$ s. In our study, the repetitions were carried out as fast as possible, and during testing we used free weights (barbell).

In our study, we chose the HVST protocol because movement velocity is a fundamental component of the intensity of resistance training. Specifically, at a given percentage of 1RM, the velocity at which a bench press is performed would influence the training effect, with the force generated during the bench press being higher when the exercise is performed at the maximal intended velocity (Seidel et al., 2015). Also, Gonzales-Badillo et al. (2014) observed that performing every repetition at maximal velocity compared to intentionally slower velocity resulted in considerably greater gains in 1RM strength and speed developed against any given load. The importance of lifting loads at maximal velocity is a key stimulus to achieve functional adaptations directed towards improving neuromuscular performance. Performing the task with maximal velocity causes greater activation of agonist muscle groups with higher peak forces attained in each repetition (Hatfield et al., 2006; Sakamoto and Sinclair, 2012). Additionally, Padulo et al. (2012) suggest that HVST is necessary to increase muscle strength among individuals with long resistance training experience. 
The non-significant main effect for groups found in our study for both movement velocity and strength endurance has practical implications not only for sport, but for rehabilitation as well. It was shown that experienced $\mathrm{CP}$ powerlifters, despite neurological deficits described earlier, could gain comparable performance of repetitive high-velocity movements to powerlifters with unaffected motor coordination in the upper limbs. This is most likely due to the long-term participation in strength training (approximately 5 years in the $\mathrm{CP}$ group) during which cyclic movements (bench press) are repeated many times. It was found that repetitive strength training methods increase movement velocity in individuals with $\mathrm{CP}$ in a reaching task (Kim et al., 2012). This may explain why there were no significant differences in movement velocity and strength endurance between $\mathrm{CP}$ and $\mathrm{CON}$ groups, both before and after the intervention.

This study had some limitations. First of all, there was no control group using traditional strength training, which would confirm the

\section{References}

Bakeman R. Recommended effect size statistics for repeated measures designs. Behav Res Methods, 2005; 37: 379-384

Beckman E, Connick M, Tweedy S. Assessing muscle strength for the purpose of classification in Paralympic sport: A review and recommendations. J Sci Med Sport, 2017; 20: 391-396

Chiu HC, Ada L, Butler J, Coulson S. Relative contribution of motor impairments to limitations in activity and restrictions in participation in adults with hemiplegic cerebral palsy. Clin Rehabil, 2010; 24: 454-462

Eken MM, Dallmeijer AJ, Doorenbosch CA, Dekkers H, Becher JG, Houdijk H. Assessment of muscle endurance of the knee extensor muscles in adolescents with spastic cerebral palsy using a submaximal repetitions-to-fatigue protocol. Arch Phys Med Rehabil, 2014; 95: 1888-1894

Eken MM, Harlaar J, Dallmeijer AJ, de Waard E, van Bennekom CM, Houdijk H. Squat test performance and execution in children with and without cerebral palsy. Clin Biomech, 2017; 41: 98-105

González-Badillo JJ, Rodríguez-Rosell D, Sánchez-Medina L, Gorostiaga EM, Pareja-Blanco F. Maximal intended velocity training induces greater gains in bench press performance than deliberately slower half-velocity training. Eur J Sport Sci, 2014; 14: 772-781

Hatfield DL, Kraemer WJ, Spiering BA, Häkkinen K. The impact of velocity of movement on performance factors in resistance exercise. J Strength Cond Res, 2006; 20: 760-766

Hussain AW, Onambele GL, Williams AG, Morse CI. Muscle size, activation, and coactivation in adults with cerebral palsy. Muscle Nerve, 2014; 49: 76-83

Kim DA, Lee JA, Hwang PW, Lee MJ, Kim HK, Park JJ, You JH, Lee DR, Lee NG. The effect of comprehensive hand repetitive intensive strength training (CHRIST) using motion analysis in children with cerebral palsy. Ann Rehabil Med, 2012; 36: 39-46

Klenck C, Gebke K. Practical management: common medical problems in disabled athletes. Clin J Sport Med, 2007; 17: 55-60

Kristiansen M, Samani A, Madeleine P, Hansen EA. Effects of 5 weeks of bench press training on muscle synergies: a randomized controlled study. J Strength Cond Res, 2016; 30: 1948-1959

Moreau NG, Holthaus K, Marlow N. Differential adaptations of muscle architecture to high-velocity versus traditional strength training in cerebral palsy. Neurorehabil Neural Repair, 2013; 27: 325-334

(c) Editorial Committee of Journal of Human Kinetics 
Padulo J, Mignogna P, Mignardi S, Tonni F, D'ottavio S. Effect of different pushing speeds on bench press. Int J Sports Med, 2012; 33: 376-380

Ritti-Dias RM, Avelar A, Salvador EP, Cyrino ES. Influence of previous experience on resistance training on reliability of one-repetition maximum test. J Strength Cond Res, 2011; 25: 1418-1422

Runciman P, Derman W, Ferreira S, Albertus-Kajee Y, Tucker R. A descriptive comparison of sprint cycling performance and neuromuscular characteristics in able-bodied athletes and paralympic athletes with cerebral palsy. Am J Phys Med Rehabil, 2015; 94: 28-37

Sakamoto A, Sinclair PJ. Muscle activations under varying lifting speeds and intensities during bench press. Eur J Appl Physiol, 2012; 112: 1015-1025

Schiariti V, Selb M, Cieza A, O'donnell M. International Classification of Functioning, Disability and Health Core Sets for children and youth with cerebral palsy: a consensus meeting. Dev Med Child Neurol, 2015; 57: $149-158$

Seidel W, Szafraniec R, Chromik K. Selected kinematics characteristic during bench press by disabled powerlifting athletes. Arch Budo Sci Martial Arts Extrem Sports, 2015; 11: 115-122

\section{Corresponding author:}

\section{Rafał Szafraniec}

Department of Paralympic Sports,

University School of Physical Education in Wroclaw, Poland,

al. Paderewskiego 35, 51-612 Wrocław

E-mail: rafal.szafraniec@awf.wroc.pl 